\title{
Unit-Gamma Distortion of Bivariate Copulas
}

\author{
Jungsywan H. SePANSKI*1 \\ ${ }^{1}$ Department of Statistics, Actuarial and Data Sciences, Central Michigan University, Mount Pleasant, \\ Michigan, USA
}

\begin{abstract}
In this paper, we advance new families of bivariate copulas constructed by distributional distortions of existing bivariate copulas. The distortions under consideration are based on the unit gamma distribution of two forms. When the initial copula is Archimedean, the induced copula is also Archimedean under the admissible parameter space. Properties such as Kendall's tau coefficient, tail dependence coefficients and tail orders for the new families of copulas are derived. An empirical application to economic indicator data is presented.
\end{abstract}

Keywords Archimedean copula; Clayton copula; distortion; Frank copula; Gumbel copula; Kendall's tau; tail dependence; tail order; unit gamma distribution

\section{Introduction}

Copulas have been employed to study the extent of tail dependence in the context of risk management, finance and insurance. The upper tail or extremal dependence refers to the degree of dependence in the upper right tail behavior and is generally defined to be the conditional probability of a vector of multivariate random variables exceeding a large threshold given that a subset of the random vector already exceeds the threshold. It is of interest in practice, for example, in modeling the dependence of large loss events among various assets or insurance policies. In this article, copula models are employed to analyze economic data. Methods for generating new families of distributions, such as the one presented here, have been investigated to obtain better fit to the data and more accommodating properties like concordance correlation coefficients, tail dependencies and a wide range of associations.

For expository purposes, we first briefly review bivariate copulas. Let $F(x, y)$ be the cumulative probability distribution (cdf) of a bivariate random vector $(X, Y), F_{X}(x)$ and $F_{Y}(y)$ be the respective marginal cdf's of $X$ and $Y$. Sklar (1959) shows that there exists a unique copula $C$, a joint cdf of uniformly distributed random variables on $[0,1] \times[0,1]$, such that

$$
F(x, y)=P(X \leq x, Y \leq y)=C\left(F_{X}(x), F_{Y}(y)\right)
$$

The function $C$ is the cdf of $U=F_{X}(x)$ and $V=F_{Y}(y)$ given by, for $0 \leq u, v \leq 1$,

$$
C(u, v)=F\left(F_{X}^{-1}(u), F_{Y}^{-1}(v)\right)
$$

where $F_{X}^{-1}$ and $F_{Y}^{-1}(v)$ are the respective quantile functions of $X$ and $Y$. The joint probability density function (pdf), denoted by $f(x, y)$, of $(X, Y)$ is

$$
f(x, y)=c\left(F_{X}(x), F_{Y}(y)\right) f_{X}(x) f_{Y}(y)
$$

*Email: sepan1jh@cmich.edu. 
where $f_{X}$ and $f_{Y}$ are the respective pdf's of $X$ and $Y$ and $c(u, v)=\partial^{2} C(u, v) / \partial u \partial v$ is the copula pdf. The relation in Equation (1) indicates that the copula pdf $c$, containing dependence information between $X$ and $Y$, links the joint pdf of $(X, Y)$ and the product of marginals, which is the joint pdf under the independence assumption.

A copula $\mathrm{C}$ is called Archimedean if it admits the representation

$$
C(u, v)=\phi^{-1}(\phi(u)+\phi(v))
$$

where the generator function $\phi:[0,1] \rightarrow[0, \infty)$ has continuous derivatives on $(0,1)$ such that $\phi(1)=0, \phi^{\prime}(t)<0$ and $\phi^{\prime \prime}(t)>0$ for $0<t<1$. Let $\phi(0)=\infty$ in the sense that $\lim _{t \rightarrow 0+} \phi(t)=\infty$. If $\phi(0)=\infty$, the generator is said to be a strict generator. These conditions ensure that $\phi^{-1}$ exists. If $\phi(0)<\infty$, the generator is not strict. In the bivariate case, an Archimedean copula of the form $C(u, v)=\max \left\{\phi^{[-1]}(\phi(u)+\phi(v)), 0\right\}$ may be obtained with weaker conditions on the generator $\phi$ and its pseudo-inverse $\phi^{[-1]}$ defined as $\phi^{-1}(t)$ if $0 \leq \phi(t) \leq \phi(0)$, and 0 if $\phi(0) \leq \phi(t)<\infty$. Note if $\phi(0)=\infty$, then $\phi^{[-1]}=\phi^{-1}$.

A function $T:[0,1] \rightarrow[0,1]$ is said to be a distortion function if it is continuous and nondecreasing, not necessarily convex or concave, with $T(0)=0$ and $T(1)=1$. A distortion $T$ is said to be admissible for an initial copula $C$ if the copula $C_{T}(u, v)$ define by

$$
C_{T}(u, v)=T\left(C\left(T^{-1}(u), T^{-1}(v)\right)\right) \text { for } u, v \in[0,1]
$$

is also a copula. From Theorem 3.3.3 in Nelsen (2006), $C_{T}(u, v)$ is a copula if only if $T$ is convex. If the initial $C$ is Archimedean with a generator $\phi$, then the new copula is Archimedean with generator $\phi \circ T^{-1}$; see the right composition rule by Genest et al. (1995) and Morillas (2005) imparted below.

Multi-parametric models would allow more flexibility and improve the fit when applied to data fitting. As pointed out by Di Bernardino and Rullière (2013), using parametric transformations or distortions of existing copulas has several possible advantages, e.g., improving both the fit to observed data by adding parameters and the convergence of estimation algorithm. Valdez and Xiao (2011) put forth three kinds of distortions of copulas, in which the distortion is applied to the copula or to the marginals or to both the copula and the marginals. Fischer and Köck (2010) present a general construction scheme of $d$-variate copulas, which generalizes the Archimedean family, study admissible conditions on the distortion functions and derive the tail dependence coefficients for power and dual power distortions. Di Bernardino and Rullière (2016) consider transformations using conversion functions and examine the impact of the transformations on tail dependence coefficients; see also references on transformations of copulas therein. Genest et al. (1998) provide four valuable rules that can be combined to construct classes of Archimedean generators from an initial generator $\phi$, including the right-composition rule stating that if $h:[0,1] \rightarrow[0,1]$ is an increasing, concave bijection, then $\phi \circ h(t)=\phi(h(t))$ generates a bivariate Archimedean copula.

The approach in this paper originates from Wirch and Hardy (1999) in which the beta distribution distortion is employed to adjust the premiums and produce a coherent risk measure in the context of risk management. The beta distortion is given by

$$
T(u)=\int_{0}^{u} \frac{1}{\mathcal{B}(\alpha, \beta)} t^{\alpha-1}(1-t)^{\beta-1} d t .
$$

where $\mathcal{B}(\alpha, \beta), \alpha, \beta>0$, is the beta function. Eugene et al. (2010) later utilize the same framework to generalize univariate cdf with $u$ replaced by any continuous cdf. The resulting cdf 
incorporates the parameters in the beta cdf and the cdf and allows more flexibility in statistical modeling. The framework has been since widely adopted and extended to generalize univariate distributions; see Alzaatreh et al. (2013) and Nadarajah and Rocha (2016) for an impressive list of literature. Making use of the framework in Equation (3), Samanthi and Sepanski (2019) propose a bivariate cdf's of a bivariate random variable $(X, Y)$ by replacing $u$ with a bivariate cdf $C$ such that

$$
H_{1}(x, y)=\int_{0}^{C\left(F_{1}(x), F_{2}(y)\right.} \frac{1}{B(\alpha, \beta)} t^{\alpha-1}(1-t)^{\beta-1} d t,
$$

where $F_{1}$ and $F_{2}$ are cdf's of continuous random variables. The new family of the bivariate cdf gives rise to a new family of copulas of the form in Equation (2).

As one can see that the cdf of a continuous random variable with a support of $[0,1]$ may serve the role of $T$ in Equation (2). A method for generating a random variable with support $[0,1]$ is to take the logarithmic transformation such that $W=\log \left(1 / X_{1}\right)$ or $W=\log \left(1 /\left(1-X_{2}\right)\right)$, where the support of $W$ is the set of non-negative real numbers. Specifically, here we hire the unit-gamma distribution functions, introduced in Grassia (1977), of the random variables $X_{1}$ and $X_{2}$ derived with $W$ being a gamma random variable. We call $X_{1}$ unit gamma I random variable and $X_{2}$ unit gamma II random variable.

This paper studies new families of bivariate copulas generated by the unit-gamma (UG) distribution distortions. It is organized as follows. The proposed new families of copulas and their properties are presented in Section 2 and Section 3. Kendall's tau coefficients are calculated for some examples. The impact of parameters in the UG distortions on Kendall's tau coefficients are explored via graphical illustrations. The tail dependence coefficients are derived for the distorted copulas. Section 4 contains an empirical application to economic indicator data, followed by conclusions in Section 5. Preliminaries are placed in the appendix.

\section{Unit Gamma I Distortion Generated Copulas}

In this section, the induced bivariate cdf, conditional cdf, and copula via UGI distortion are presented. We also study properties of the UGI generated copulas such as Kendall's tau coefficient, tail dependence coefficients and tail orders.

Let $W$ be a gamma random variable with pdf given by

$$
g(w)=\beta^{\alpha} w^{\alpha-1} e^{-\beta w} / \Gamma(\alpha), \alpha>0, \beta>0,
$$

where $\Gamma(\alpha)$ is the gamma function. A unit-gamma I (UGI) random variable $X_{1}$ is defined by $X_{1}=e^{-W}$. The respective pdf, cdf and quantile function of the UGI random variable $X_{1}$ are given by, for $0<u<1$,

$$
\begin{aligned}
& h_{1}(u)=\frac{\beta^{\alpha}}{\Gamma(\alpha)}[\log (1 / u)]^{\alpha-1} u^{\beta-1}, \\
& H_{1}(u)=\int_{0}^{u} h_{1}(x) d x=1-G(-\log u, \alpha, \beta)=1-G(-\beta \log u, \alpha, 1), \\
& H_{1}^{-1}(u)=\exp \left\{-G^{-1}(1-u, \alpha, \beta)\right\}=\exp \left\{-G^{-1}(1-u, \alpha, 1) / \beta\right\},
\end{aligned}
$$

where $G(\cdot)$ and $G^{-1}(\cdot)$ are the cdf and the quantile function of a gamma random variable with shape parameter $\alpha$ and rate parameter $\beta$. When $\alpha=1, G(w, 1, \beta)=1-\exp (-\beta w)$ for $w \geq 0$ 
and the quantile function $G^{-1}(u, 1, \beta)=-\log (1-u) / \beta$ for $0<u<1$. Setting $\alpha=1$, the $H_{1}(u)=u^{\beta}$, i.e., the power transformation.

Implementing Equation (4) with the UGI distortion leads to a joint cdf for a bivariate random variable $(X, Y)$ such that, for $-\infty<x, y<\infty$,

$$
F(x, y)=\frac{\beta^{\alpha}}{\Gamma(\alpha)} \int_{0}^{C\left(F_{1}(x), F_{2}(y)\right)}[\log (1 / u)]^{\alpha-1} u^{\beta-1} d u=H_{1}\left(C\left(F_{1}(x), F_{2}(y)\right)\right),
$$

where $F_{1}$ and $F_{2}$ are cdf's of any continuous random variables and $C(u, v)$ is an initial copula. Note that in what follows, a prime mark denotes the first derivative of a function, e.g., $F_{1}^{\prime}(x)=$ $d F(x) / d x$. The corresponding pdf is

$$
\begin{aligned}
f(x, y)=\left[h_{1}^{\prime}(\right. & \left.C\left(F_{1}(x), F_{2}(y)\right)\right) C_{2 \mid 1}\left(F_{2}(y) \mid F_{1}(x)\right) C_{1 \mid 2}\left(F_{1}(x) \mid F_{2}(y)\right) \\
& \left.+h_{1}\left(C\left(F_{1}(x), F_{2}(y)\right)\right) c\left(F_{1}(x), F_{2}(y)\right)\right] f_{1}(x) f_{2}(y)
\end{aligned}
$$

where $f_{1}(x)=F_{1}^{\prime}(x), f_{2}(y)=F_{2}^{\prime}(y), C_{2 \mid 1}=\partial C(u, v) / \partial u, C_{1 \mid 2}=\partial C(u, v) / \partial v$ and $c(u, v)=$ $\partial^{2} C(u, v) / \partial u \partial v$. The margins or marginal distributions of $X$ and $Y$ are $F_{X}(x)=H_{1}\left(F_{1}(x)\right)$ and $H_{1}\left(F_{1}(y)\right)$, respectively. If $H_{1}$ in Equation (5) is admissible, then the induced UGI distorted copula is given by

$$
C_{H_{1}}(u, v)=H_{1}\left(C\left(H_{1}^{-1}(u), H_{1}^{-1}(v)\right)\right) .
$$

As a result of Theorem 3.3.3 in Nelsen (2006) stating that $C_{H_{1}}$ in Equation (8) is a copula if and only if $H_{1}$ is convex, we derive the following.

Proposition 1. The distortion $H_{1}:[0,1] \rightarrow[0,1]$ is admissible if $0<\alpha \leq 1$ and $\beta \geq 1$.

Proof. A necessary and sufficient condition for $H_{1}$ to be convex is that its second derivative is non-negative for all $u$ in $[0,1]$. The second derivative of $H_{1}$ given by

$$
h_{1}^{\prime}(u)=\frac{\beta^{\alpha}}{\Gamma(\alpha)}[\log (1 / u)]^{\alpha-2} u^{\beta-2}[-(\alpha-1)+\log (1 / u)(\beta-1)],
$$

which is non-negative if $\beta \geq 1$ and $0<\alpha \leq 1$. If $\beta<1$, the convexity condition cannot be satisfied at any $\alpha>0$ for all $u \in[0,1]$.

The conditional cdf of the new family of copulas is given by

$$
C_{H_{1}, 2 \mid 1}(v \mid u)=\frac{\partial C_{H_{1}}(u, v)}{\partial u}=\frac{h_{1}\left(C\left(H_{1}^{-1}(u), H_{1}^{-1}(u)\right)\right)}{h_{1}\left(H_{1}^{-1}(u)\right)} C_{2 \mid 1}\left(H_{1}^{-1}(u) \mid H_{1}^{-1}(u)\right) .
$$

The copula pdf $c_{H_{1}}(u, v)$ of $C_{H_{1}}$ is given by

$$
\begin{gathered}
\frac{h_{1}^{\prime}\left(C\left(H_{1}^{-1}(u), H_{1}^{-1}(u)\right)\right)}{h_{1}\left(H_{1}^{-1}(u)\right) h_{1}\left(H_{1}^{-1}(u)\right)} C_{2 \mid 1}\left(H_{1}^{-1}(u) \mid H_{1}^{-1}(u)\right) C_{1 \mid 2}\left(H_{1}^{-1}(u) \mid H_{1}^{-1}(u)\right) \\
+\frac{h_{1}\left(C\left(H_{1}^{-1}(u), H_{1}^{-1}(u)\right)\right)}{h_{1}\left(H_{1}^{-1}(u)\right) h_{1}\left(H_{1}^{-1}(u)\right)} c\left(H_{1}^{-1}(u), H_{1}^{-1}(u)\right),
\end{gathered}
$$

where $C_{2 \mid 1}(v \mid u)=\partial C(u, v) / \partial u$ and $C_{1 \mid 2}(u \mid v)=\partial C(u, v) / \partial v$. If $H_{1}$ is admissible, $h_{1}^{\prime}$ is nonnegative and so is Equation (9). 
For a strict Archimedean copula $C$ with generator $\phi$ and $\psi=\phi^{-1}$, the conditional copula distribution $C(v \mid u)$ is given by

$$
C_{2 \mid 1}(v \mid u)=\frac{\partial C(u, v)}{\partial u}=\psi^{\prime}(\phi(u)+\phi(v)) \phi^{\prime}(u)=\left[\phi^{\prime}(\psi(\phi(u)+\phi(v)))\right]^{-1} \phi^{\prime}(u) .
$$

The conditional distribution function method expressed in three steps can be used to generate pairs of observations $(u, v)$ from $C$ :(i) generate two independent variates $u$ and $t$ from uniform $(0,1)$; (ii) set $w=\left(\phi^{\prime}\right)^{-1}\left(\phi^{\prime}(u) / t\right)$ or $w=\left(\psi^{\prime}\right)^{-1}\left(t / \phi^{\prime}(u)\right)$; and (iii) set $v=\psi(\phi(w)-\phi(u))$ or $v=\psi(w-\phi(u))$. In step (ii), one solves the equation $\psi^{\prime}(w)=t / \phi^{\prime}(u)$ for $w$. When the UGI distribution distortion is applied to an Archimedean copula $C$ with initial generator $\phi$,

$$
C_{H_{1}}(u, v)=H_{1} \circ \phi^{-1}\left(\phi\left(H^{-1}(u)\right)+\phi\left(H^{-1}(v)\right)\right) .
$$

By the right-composition rule in Genest et al. (1998), $C_{H_{1}}$ is Archimedean with generator $\phi \circ H^{-1}$ as $H^{-1}$ is increasing and concave. The first derivatives of the generator and its inverse generator to be employed for simulations are $\phi^{\prime}\left(H_{1}^{-1}(t)\right)\left(H^{-1}\right)^{\prime}(t)$ and $h_{1}(\psi(t)) \psi^{\prime}(t)$, respectively.

\subsection{Kendall's Tau Coefficient}

The formula for the calculation of Kendall's tau coefficient from a copula is in Equation (20) in the appendix. If the initial copula $C$ is Archimedean with strict generator $\phi$, then one can calculate Kendall's tau coefficient using Equation (21). In this case, Kendall's tau of $C_{H_{1}}$ is given by, with a substitution of $H_{1}^{-1}(t)=s$,

$$
\tau_{C_{H_{1}}}=1+4 \int_{0}^{1} \frac{\phi\left(H_{1}^{-1}(t)\right) H_{1}^{\prime}\left(H_{1}^{-1}(t)\right)}{\phi^{\prime}\left(H_{1}^{-1}(t)\right)} d t=1+4 \int_{0}^{1} \frac{\phi(s)}{\phi^{\prime}(s)} h_{1}^{2}(s) d s .
$$

According to Equation (10), the explicit functional form of the induced generator $\phi\left(H_{1}^{-1}(t)\right)$ is not necessary for the calculation of Kendall's tau.

Example 1 (UGI-Clayton Copula). Consider the case when the initial copula is the Clayton copula given by $C(u, v)=\left(u^{-\theta}+v^{-\theta}-1\right)^{-1 / \theta}$, where $\theta>0$. By Equation (10), Table 4 in the appendix and the fact that $\int_{0}^{1}[\log (1 / u)]^{a-1} u^{b-1} d u=b^{a} / \Gamma(a)$, Kendall's tau, $\tau_{I C}$, for the UGI-Clayton copula is

$$
\begin{aligned}
\tau_{I C} & =1-4 \int_{0}^{1} \frac{1}{\theta}\left(t-t^{\theta+1}\right) h_{1}^{2}(t) d t \\
& =1-\frac{4 \beta^{2 \alpha} \Gamma(2 \alpha-1)}{\theta[\Gamma(\alpha)]^{2}}\left[\frac{1}{(2 \beta)^{2 \alpha-1}}-\frac{1}{(2 \beta+\theta)^{2 \alpha-1}}\right] .
\end{aligned}
$$

When $\alpha=\beta=1, \tau_{I C}=\theta /(\theta+2)$. The power function distorted Clayton copula, i.e., $\alpha=1$, has a Kendall's tau value of $1-2 \beta /(2 \beta+\theta)$, which decreases as $\beta$ increases at a fix $\theta$; see also Figure 1 below.

Example 2 (UGI-Gumbel Copula). The bivariate Gumbel copula is defined as

$$
C(u, v)=\exp \left\{-\left[(-\log u)^{\theta}+(-\log v)^{\theta}\right]^{1 / \theta}\right\},
$$

where $\theta \geq 1$. From Equation (5) and (6),

$$
H_{1}\left(C\left(H_{1}^{-1}(u), H_{1}^{-1}(v)\right)\right)=1-G\left(\left(\left[G^{-1}(1-u, \alpha, 1)\right]^{\theta}+\left[G^{-1}(1-v, \alpha, 1)\right]^{\theta}\right)^{1 / \theta}, \alpha, 1\right) .
$$


The parameter $\beta$ in the UGI distribution vanishes in the UGI-Gumbel copula. Note also that the Gumbel copula is an extreme-value copula such that $C(u, v)=C^{n}\left(u^{1 / n}, v^{1 / n}\right)$ for all $n>0$. When $\alpha=1$, the UGI distortion is the power transformation and thus returns the Gumbel copula.

Kendall's tau, $\tau_{I G}$, of the UGI-Gumbel copula is given by

$$
\tau_{I G}=1-\frac{4 \beta^{2 \alpha}}{\theta[\Gamma(\alpha)]^{2}} \int_{0}^{1}[-\log (t)]^{2 \alpha-1} t^{2 \beta-1} d t=1-\frac{4 \Gamma(2 \alpha)}{2^{2 \alpha} \theta[\Gamma(\alpha)]^{2}},
$$

in which, as explained above, the parameter $\beta$ doesn't play a role. The $\tau_{I G}$ value falls between $1-1 / \theta$ and 1 since $\Gamma(2 \alpha) /\left\{2^{2 \alpha}[\Gamma(\alpha)]^{2}\right\}$ is an increasing function of $\alpha$ and has a limit of 0 as $\alpha \rightarrow 0$.

Example 3 (UGI-Frank Copula). The Frank copula is defined as

$$
C(u, v)=-\frac{1}{\theta} \log \left(1+\frac{\left(e^{-\theta u}-1\right)\left(e^{-\theta v}-1\right)}{\left(e^{-\theta}-1\right)}\right)
$$

where $\theta \neq 0$. Let $k_{1}(t)=\int_{0}^{t}\left(e^{\theta u}-1\right)\left[h_{1}(u)\right]^{2} d u$. Note that $k_{1}(0)=0$. From Table 4 in the appendix, the integral $\int_{0}^{1} \phi(t) / \phi^{\prime}(t)\left[h_{1}(t)^{2}\right] d t$ in Equation (10) for the UG1-Frank copula is given by, applying integration by parts,

$$
\begin{aligned}
& \frac{1}{\theta} \int_{0}^{1}\left(e^{\theta t}-1\right) \log \left(\frac{e^{-\theta t}-1}{e^{-\theta}-1}\right)\left[h_{1}(t)\right]^{2} d t \\
= & \frac{1}{\theta}\left\{\left.\left[\log \left(e^{-\theta t}-1\right)-\log \left(e^{-\theta}-1\right)\right] k_{1}(t)\right|_{t=0} ^{1}-\int_{0}^{1} f_{1}(t) \frac{\theta}{e^{\theta t}-1} d t\right\} \\
= & -\int_{0}^{1} \frac{1}{e^{\theta t}-1} \int_{0}^{t}\left(e^{\theta u}-1\right)\left[h_{1}(u)\right]^{2} d u d t .
\end{aligned}
$$

When $\alpha=\beta=1$, then Equation (11) is equal to $-1 / \theta+\int_{0}^{1} t /\left(e^{\theta t}-1\right) d t=-1 / \theta+\theta^{-2} \int_{0}^{\theta} t /\left(e^{t}-1\right) d t$. The formula in Equation (11) is used to numerically compute Kendall's tau the UGI-Frank copula below.

To examine the impact of the proposed distortions on Kendall's tau, we numerically computed Kendall's tau as a function of the parameters $\alpha$ and $\beta$ at $|\theta|=4$ and constructed threedimensional tau plots. The last column of Figure 1 is the tau plot for the distorted Frank copula with $\theta=-4$.

In general, as shown in Figure 1, Kendall's tau of the UGI distorted copula increases as $\alpha$ decreases. The parameter $\beta$ is not involved in the UGI-Gumbel copula family as explained in Example 2 and hence doesn't affect tau values. For the UGI-Frank copula, Kendall's tau decreases as $\beta$ increases when $\theta=4$ and increases as $\beta$ increases with minimum occurred at $\alpha=1$ and $\beta=1$ when $\theta=-4$.

\subsection{Tail Dependence Coefficients}

Consider next the tail dependence coefficients for the new family of copulas based on the UGI distortion, where $0<\alpha \leq 1$ and $\beta \geq 1$. Definitions of upper and lower tail dependence coefficients are presented in Equation (22) in the appendix. 
Clayton

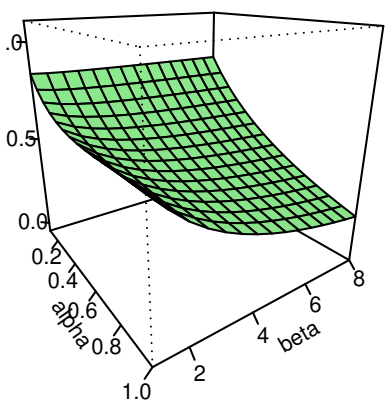

Gumbe

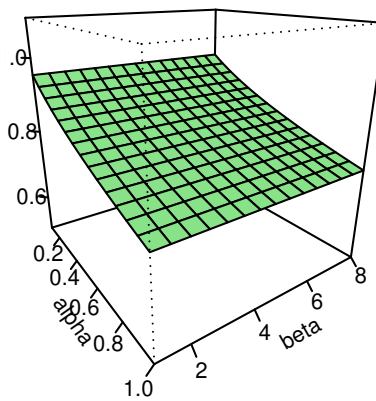

Frank

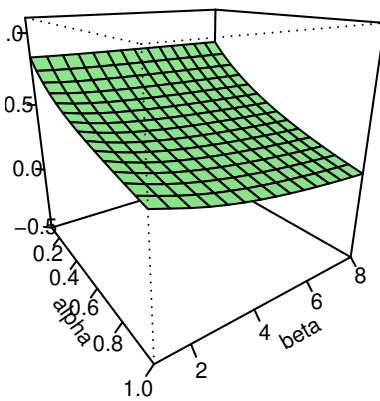

Frank

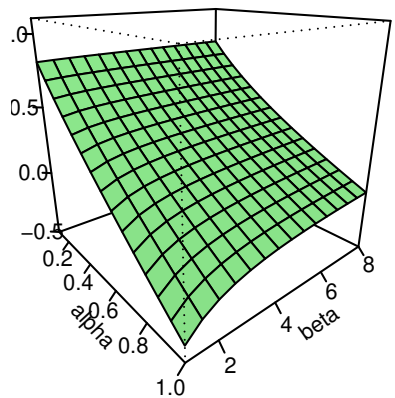

Figure 1: Kendall's tau of UGI distorted copulas with the initial copula specified in the title with $|\theta|=4$ for $\alpha$ (alpha) $\in(0.1,1]$ and $\beta$ (beta) $\in[1,8]$.

Proposition 2. Let $\lambda_{L}$ and $\lambda_{U}$ be the finite lower tail and upper tail dependence coefficients of the initial copula $C$. Then the UGI induced copula $C_{H_{1}}$ has a lower tail dependence coefficient $\lambda_{L, H_{1}}=\left(\lambda_{L}\right)^{\beta}$ and an upper tail dependence coefficient $\lambda_{U, H_{1}}=2-\left(2-\lambda_{U}\right)^{\alpha}$.

Proof. By Equation (8) and (22) and L'Hospital's Rule, the lower tail dependence coefficient is

$$
\begin{aligned}
\lambda_{L, H_{1}} & =\lim _{u \rightarrow 0+} \frac{H_{1}\left(C\left(H_{1}^{-1}(u), H_{1}^{-1}(u)\right)\right.}{u} \\
& =\lim _{u \rightarrow 0} \frac{H_{1}(C(u, u))}{H_{1}(u)}=\lim _{u \rightarrow 0+} \frac{h_{1}(C(u, u))}{h_{1}(u)} \frac{d C(u, u)}{d u} \\
& =\lim _{u \rightarrow 0+}\left[\frac{-\log (C(u, u))}{-\log (u)}\right]^{\alpha-1}\left[\frac{C(u, u)}{u}\right]^{\beta-1} \frac{d C(u, u)}{d u} \\
& =\lim _{u \rightarrow 0+}\left[\frac{u}{C(u, u)} \frac{d C(u, u)}{d u}\right]^{\alpha-1} \lim _{u \rightarrow 0+}\left[\frac{C(u, u)}{u}\right]^{\beta-1} \lim _{u \rightarrow 0+} \frac{d C(u, u)}{d u} \\
& =\left(\lambda_{L}\right)^{\beta} .
\end{aligned}
$$

Note $0<\alpha \leq 1$ and $-\log (u) /[-\log (C(u, u))]$ is bounded by 1 since $C(u, u) \leq u$. If $\lambda_{L}=\lim _{u \rightarrow 0} C(u, u) / u=0$, the limit in Equation (12) is 0. The upper tail dependence coefficient is given by

$$
\begin{aligned}
\lambda_{U, H_{1}} & =2-\lim _{u \rightarrow 1-} \frac{1-H_{1}\left(C\left(H_{1}^{-1}(u), H_{1}^{-1}(u)\right)\right.}{1-u} \\
& =2-\lim _{u \rightarrow 1-} \frac{1-H_{1}(C(u, u))}{1-H_{1}(u)}=2-\lim _{u \rightarrow 1-} \frac{h_{1}(C(u, u))}{h_{1}(u)} \frac{d C(u, u)}{d u} \\
& =2-\lim _{u \rightarrow 1-}\left[\frac{u}{C(u, u)} \frac{d C(u, u)}{d u}\right]^{\alpha-1} \lim _{u \rightarrow 1-}\left[\frac{C(u, u)}{u}\right]^{\beta-1} \lim _{u \rightarrow 1-} \frac{d C(u, u)}{d u} \\
& =2-\left(2-\lambda_{U}\right)^{\alpha},
\end{aligned}
$$

since $\lim _{u \rightarrow 1-} C(u, u) / u=1$ and $d C(u, u) / d u=2-\lambda_{U}$. 


\subsection{Tail Orders}

In this section, we study tail orders of the copula $C_{H_{1}}$. Definitions of tail orders can be found in the appendix, and we note here only the joint survival function $\bar{C}(u, v)=P(U>u, V>v)=$ $1-u-v+C(u, v)$.

Proposition 3. Suppose that $C(u, u) \sim u^{\kappa_{L}} \ell(u)$ as $u \rightarrow 0^{+}$and $\bar{C}(1-u, 1-u) \sim u^{\kappa_{U}} \ell_{*}(u)$ as $u \rightarrow 0+$ for some slowly varying functions $\ell$ and $\ell_{*}$ at $0+$. Then $C_{H_{1}}$ has a lower tail order $\kappa_{L, H_{1}}=\kappa_{L}$ and an upper tail order $\kappa_{U, H_{1}}=1$.

Proof. The function $\log (1 / u)$ is slowly varying at $u=0+$, by Theorem A1 in the appendix, $h_{1}(u)$ is regularly varying with index $\beta-1$ at $u=0+$. By Theorem A3, $H_{1}(u)=\int_{0}^{u} h_{1}(t) d t$ is regularly varying with index $\beta$, where $\beta \geq 1$. Let $\ell_{H_{1}}$ be the associated slowly varying function for $H_{1}$, then as $u \rightarrow 0+$,

$$
\begin{aligned}
H_{1}\left(C\left(H_{1}^{-1}(u), H_{1}^{-1}(u)\right)\right. & \sim\left[C\left(H_{1}^{-1}(u), H_{1}^{-1}(u)\right)\right]^{\beta} \ell_{H_{1}}\left(C\left(H_{1}^{-1}(u), H_{1}^{-1}(u)\right)\right) \\
& \sim\left[H^{-1}(u)\right]^{\beta \kappa_{L}} \ell\left(H_{1}^{-1}(u)\right) \ell_{H_{1}}\left(C\left(H_{1}^{-1}(u), H_{1}^{-1}(u)\right)\right) .
\end{aligned}
$$

Applying Theorem A4 and Theorem A2 in Appendix $5, H^{-1}$ is regularly varying with index $1 / \beta$ and $\ell(v) * \ell_{H_{1}}(C(v, v))$ is slowly varying at $v=0+$. Therefore, the lower tail order of a UGI induced copula remains the same as the one of the initial copula.

Since $-\log (1-v)=v+O\left(v^{2}\right)$ for $|v|<1$, we have that

$$
\begin{aligned}
\lim _{v \rightarrow 0+} \frac{1-H_{1}(1-v)}{v^{\alpha}} & =\lim _{v \rightarrow 0+} \frac{h_{1}(1-v)}{\alpha v^{\alpha-1}}=\lim _{v \rightarrow 0+} \frac{\beta^{\alpha}[-\log (1-v)]^{\alpha-1}(1-v)^{\beta-1}}{\alpha \Gamma(\alpha) v^{\alpha-1}} \\
& =\lim _{v \rightarrow 0+} \frac{\beta^{\alpha}\left[v+O\left(v^{2}\right)\right]^{\alpha-1}(1-v)^{\beta-1}}{\alpha v^{\alpha-1} \Gamma(\alpha)}=\frac{\beta^{\alpha}}{\alpha \Gamma(\alpha)}
\end{aligned}
$$

That is, $1-H_{1}(1-v) \sim a_{1} v^{\alpha}$ as $v \rightarrow 0+$, where $a_{1}=\beta^{\alpha} /[\alpha \Gamma(\alpha)]$. Let $\bar{C}_{H_{1}}$ be the joint survival function associated with $C_{H_{1}}$ in Equation (8). Since $\bar{C}_{H_{1}}\left(H_{1}(1-v), H_{1}(1-v)\right)=$ $2\left[1-H_{1}(1-v)\right]-\left[1-H_{1}(C(1-v, 1-v))\right]$ and $1-H_{1}(C(1-v, 1-v)) \sim a_{1}[1-C(1-v, 1-v)]^{\alpha}$ from Equation (14), as $v \rightarrow 0+$,

$$
\begin{aligned}
& \lim _{u \rightarrow 0^{+}} \frac{\bar{C}_{H_{1}}(1-u, 1-u)}{u}=\lim _{v \rightarrow 0^{+}} \frac{\bar{C}_{H_{1}}\left(H_{1}(1-v), H_{1}(1-v)\right)}{1-H_{1}(1-v)} \\
= & 2-\lim _{v \rightarrow 0^{+}} \frac{a_{1}[1-C(1-v, 1-v)]^{\alpha}}{1-H_{1}(1-v)}=2-\lim _{v \rightarrow 0^{+}} \frac{a_{1} v^{\alpha}[2-\bar{C}(1-v, 1-v) / v]^{\alpha}}{1-H_{1}(1-v)} \\
= & 2-\left[2-\lim _{v \rightarrow 0+} \frac{\bar{C}(1-v, 1-v)}{v}\right]^{\alpha}
\end{aligned}
$$

since $1-C(1-v, 1-v)=2 v-\bar{C}(1-v, 1-v)$. Therefore, $\kappa_{U, H_{1}}=1$. The results also further verify Proposition 2.

\section{Unit Gamma II Distortion Generated Copulas}

In the section, we introduce the UGII distortion and investigate Kendall's tau coefficient, tail dependence coefficients and tail orders of the resulting family of copulas. 
A unit-gamma II random variable $X_{2}$ is defined by $X_{2}=1-e^{-W}$, where $W$ is a gamma random variable. Its pdf, cdf and quantile function are given by, respectively, for $0<u<1$,

$$
\begin{aligned}
h_{2}(u) & =\frac{\beta^{\alpha}}{\Gamma(\alpha)}[\log (1 /(1-u))]^{\alpha-1}(1-u)^{\beta-1}, \\
H_{2}(u) & =\int_{0}^{u} h_{2}(x) d x=G(-\log (1-u), \alpha, \beta), \\
H_{2}^{-1}(u) & =1-\exp \left\{-G^{-1}(u, \alpha, \beta)\right\} .
\end{aligned}
$$

From Equation (5), $H_{2}(1-u)=1-H_{1}(u)$. Setting $\alpha=1, H_{2}(u)=1-(1-u)^{\beta}$, the dual power transformation.

The UGII-copula bivariate random variable $(X, Y)$ has joint cdf given by

$$
H_{2}\left(C\left(F_{1}(x), F_{2}(y)\right)\right)=\frac{\beta^{\alpha}}{\Gamma(\alpha)} \int_{0}^{C\left(F_{1}(x), F_{2}(y)\right)}\left[\log \left(\frac{1}{1-u}\right)\right]^{\alpha-1}(1-u)^{\beta-1} d u
$$

where $F_{1}$ and $F_{2}$ are cdf's of any continuous random variables and $C(u, v)$ is an initial copula. If $\mathrm{H}_{2}$ is admissible, then the new family of copulas generated by UG II distribution function is

$$
C_{H_{2}}(u, v)=H_{2}\left(C\left(H_{2}^{-1}(u), H_{2}^{-1}(v)\right)\right) .
$$

The corresponding pdf, conditional pdf and copula density function can be easily derived with $H_{1}$ and $h_{1}$ replaced by $H_{2}$ and $h_{2}$ in Equation (7) and (9).

The following proposition is shown using the Theorem 3.3.3 in Nelsen (2006).

Proposition 4. The distortion $H_{2}:[0,1] \rightarrow[0,1]$ is admissible if $\alpha \geq 1$ and $0<\beta \leq 1$.

Proof. The second derivative of $H_{2}$ is given by, for $0<u<1$,

$$
h_{2}^{\prime}(u)=\frac{\left.\beta^{\alpha} \log (1 /(1-u))\right]^{\alpha-2}(1-u)^{\beta-2}}{\Gamma(\alpha)}\left[(\alpha-1)-\log \left(\frac{1}{1-u}\right)(\beta-1)\right] .
$$

The convexity of the distortion $H_{2}$ requires that $[(\alpha-1)+\log (1-u)(\beta-1)] \geq 0$ for all $0<$ $u<1$. When $\beta \leq 1$, the convexity condition is met if $\alpha \geq 1$. When $\beta>1$, it cannot be satisfied at any finite $\alpha>0$ for all $0<u<1$.

\subsection{Kendall's Tau Coefficient}

Kendall's tau coefficients are calculated for the UGII-Clayton and UGII-Gumbel copulas. Kendall's tau for UGII-Frank has no closed form and can be numerically computed using Equation (11) with the pdf $h_{1}$ replaced by $h_{2}$.

Example 4 (UGII-Clayton Copula). By (10), Table 4 in Appendix 5 and a substitution of $s=-\log (1-t)$,

$$
\begin{aligned}
& \int_{0}^{\infty} t^{b}[-\log (1-t)]^{\alpha-1}(1-t)^{\beta-1} d t=\int_{0}^{\infty}\left(1-e^{-s}\right)^{b} s^{\alpha-1} e^{-\beta s} d s \\
& =\sum_{i=0}^{\infty}\left(\begin{array}{l}
b \\
i
\end{array}\right) \int_{0}^{\infty}\left(-e^{-s}\right)^{i} s^{\alpha-1} e^{-\beta s} d s=\sum_{i=0}^{\infty}\left(\begin{array}{l}
b \\
i
\end{array}\right) \frac{(-1)^{i} \Gamma(\alpha)}{(\beta+i)^{\alpha}}
\end{aligned}
$$


since for $b>0,\left(1-e^{-s}\right)^{b}=\sum_{i=0}^{\infty}\left(\begin{array}{l}b \\ i\end{array}\right)\left(-e^{-s}\right)^{i}$ for $\left|e^{-s}\right|<1$. By Equation (21), Kendall's tau, $\tau_{I I C}$, of the UGII-Clayton copula is equal to

$$
\begin{aligned}
\tau_{\text {IIC }}=1-\frac{4 \beta^{2 \alpha}}{[\Gamma(\alpha)]^{2}} \int_{0}^{1} \frac{t-t^{\theta+1}}{\theta}[-\log (1-t)]^{2 \alpha-2}(1-t)^{2 \beta-2} d t \\
=1-\frac{4 \beta^{2 \alpha} \Gamma(2 \alpha-1)}{\theta[\Gamma(\alpha)]^{2}}\left[\frac{1}{(2 \beta-1)^{2 \alpha-1}}-\frac{1}{(2 \beta)^{2 \alpha-1}}\right. \\
\left.-\sum_{i=0}^{\infty}\left(\begin{array}{c}
\theta+1 \\
i
\end{array}\right) \frac{(-1)^{i}}{(2 \beta+i-1)^{2 \alpha-1}}\right] .
\end{aligned}
$$

When $\alpha=\beta=1$, by the Taylor expansion of $(1-x)^{\theta+1}$ about $0, \tau_{I I G}=\theta /(\theta+2)$ since $\int_{0}^{1}(1-x)^{\theta+1} d x=\sum_{i=0}^{\infty}\left(\begin{array}{c}\theta+1 \\ i\end{array}\right)(-1)^{i}(i+1)^{-1}=1 /(\theta+2)$.

Example 5 (UGII-Gumbel Copula). Unlike the UGI-Gumbel copula in Example 2, the parameter $\beta$ stays to play a role in the induced UGII-Gumbel copula. Since

$$
\frac{\Gamma\left(\gamma_{1}\right)}{\left(\gamma_{2}+1\right)^{\gamma_{1}}}=\int_{0}^{1}(1-t)[-\log (1-t)]^{\gamma_{1}-1}(1-t)^{\gamma_{2}} d t
$$

for $\gamma_{1}>0$ and $\gamma_{2}>0$ and $\log (t)=-\sum_{i=1}^{\infty}(1-t)^{i} / i$ for $|1-t|<1$, Kendall's tau, $\tau_{I I G}$, of the UGII-Gumbel copula is

$$
\begin{aligned}
\tau_{I I G} & =1-\frac{4 \beta^{2 \alpha}}{\theta[\Gamma(\alpha)]^{2}} \int_{0}^{1} t[-\log (1-t)]^{2 \alpha-2}(1-t)^{2 \beta-2} \sum_{i=1}^{\infty} \frac{(1-t)^{i}}{i} d t \\
& =1-\frac{4 \beta^{2 \alpha} \Gamma(2 \alpha-1)}{\theta[\Gamma(\alpha)]^{2}} \sum_{i \geq 1} \frac{1}{i}\left[\frac{1}{(2 \beta+i-1)^{2 \alpha-1}}-\frac{1}{(2 \beta+i)^{2 \alpha-1}}\right] .
\end{aligned}
$$

When $\alpha=\beta=1, \tau_{I I G}=1-1 / \theta$ since $\sum_{i=1}^{\infty} 1 /[i(i+1)(i+2)]=1 / 4$.

Kendall's tau plots in Figure 2 are constructed for the same parameter values used in Figure 1. From Figure 2, one can see that with initial copulas of Clayton, Gumbel, and Frank copulas, Kendall's tau of the UGII induced copulas increases as $\beta$ decreases. Similar to the UGI distortion, the UGII-Frank copula appears to have a minimum tau value at $\alpha=\beta=1$. Same patterns appear to be present at other $\theta$ values for both the UGI and UGII distortions, though the graphs are not included here.

\subsection{Tail Dependence Coefficients}

Consider next the tail dependence coefficients of the UGII distorted copulas. Note that the admissible parameter space of $H_{2}$ is $\alpha \geq 1$ and $0<\beta \leq 1$.

Proposition 5. Let $\lambda_{L}$ and $\lambda_{U}$ be the finite lower tail and upper tail dependence coefficients of the initial copula $C$. Then the UGII distortion generated copula $C_{H_{2}}$ has a lower tail dependence coefficient $\lambda_{L, H_{2}}=\left(\lambda_{L}\right)^{\alpha}$ and an upper tail dependence coefficient $\lambda_{U, H_{2}}=2-\left(2-\lambda_{U}\right)^{\beta}$.

Proof. Similar to the derivations in Proposition 2, the lower tail dependence coefficient of the family of $\mathrm{H}_{2}$ distortion generated copulas is given by,

$$
\lambda_{L, H_{2}}=\lim _{u \rightarrow 0+} \frac{H_{2}\left(C\left(H_{2}^{-1}(u), H_{2}^{-1}(u)\right)\right.}{u}=\lim _{u \rightarrow 0+} \frac{h_{2}(C(u, u))}{h_{2}(u)} \frac{d C(u, u)}{d u}
$$


Clayton

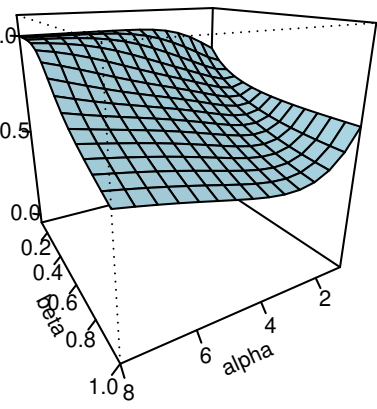

Gumbe

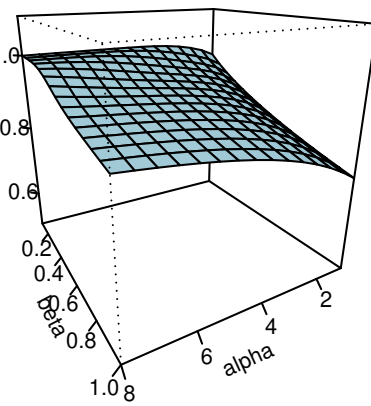

Frank

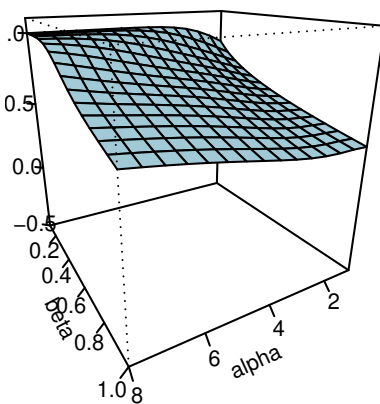

Frank

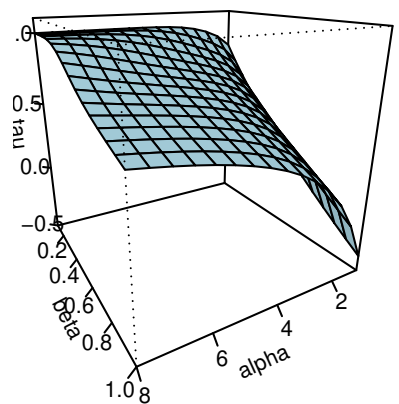

Figure 2: Kendall's tau of UGII distorted copulas with the initial copula specified in the title with $|\theta|=4$ for $\alpha$ (alpha) $\in[1,8]$ and $\beta$ (beta) $\in[0.1,1]$.

$$
\begin{aligned}
& =\lim _{u \rightarrow 0+}\left[\frac{-\log (1-C(u, u))}{-\log (1-u)}\right]^{\alpha-1}\left[\frac{1-C(u, u)}{1-u}\right]^{\beta-1} \frac{d C(u, u)}{d u} \\
& =\lim _{u \rightarrow 0+}\left[\frac{1-u}{1-C(u, u)} \frac{d C(u, u)}{d u}\right]^{\alpha-1} \lim _{u \rightarrow 0+}\left[\frac{1-C(u, u)}{1-u}\right]^{\beta-1} \lim _{u \rightarrow 0+} \frac{d C(u, u)}{d u} \\
& =\left(\lambda_{L}\right)^{\alpha} .
\end{aligned}
$$

And the upper tail dependence coefficient is given by

$$
\begin{aligned}
\lambda_{U, H_{2}} & =2-\lim _{u \rightarrow 1-} \frac{1-H_{2}\left(C\left(H_{2}^{-1}(u), H_{2}^{-1}(u)\right)\right.}{1-u} \\
& =2-\lim _{u \rightarrow 1-}\left[\frac{-\log (1-C(u, u))}{-\log (1-u)}\right]^{\alpha-1}\left[\frac{1-C(u, u)}{1-u}\right]^{\beta-1} \frac{d C(u, u)}{d u} \\
& =2-\lim _{u \rightarrow 1-}\left[\frac{1-u}{1-C(u, u)} \frac{d C(u, u)}{d u}\right]^{\alpha-1} \lim _{u \rightarrow 1-}\left[\frac{d C(u, u)}{d u}\right]^{\beta-1} \lim _{u \rightarrow 1-} \frac{d C(u, u)}{d u} \\
& =2-\left(2-\lambda_{U}\right)^{\beta}
\end{aligned}
$$

Note the term $[-\log (1-C(u, u))] /[-\log (1-u)] /$ in Equation (17) is bounded by 1 , and if $\lim _{u \rightarrow 1-}[1-C(u, u)] /(1-u)=0$, then Equation (17) gives that $\lambda_{U, H_{2}}=2$.

\subsection{Tail Orders}

Proposition 6. Suppose that $C(u, u) \sim u^{\kappa_{L}} \ell(u)$ as $u \rightarrow 0^{+}$and $\bar{C}(1-u, 1-u) \sim u^{\kappa_{U}} \ell_{*}(u)$ as $u \rightarrow 0+$ for some slowly varying functions $\ell$ and $\ell_{*}$ at $0+$. Then $C_{H_{2}}$ has a lower tail order $\kappa_{L, H_{2}}=\kappa_{L}$ and an upper tail order $\kappa_{U, H_{2}}=1$.

Proof. Since $-\log (1-u)=u+O\left(u^{2}\right)$ for $|u|<1$,

$$
\lim _{u \rightarrow 0+} \frac{H_{2}(u)}{u^{\alpha}}=\lim _{u \rightarrow 0+} \frac{h_{2}(u)}{\alpha u^{\alpha-1}}=\lim _{u \rightarrow 0+} \frac{\beta^{\alpha}\left[u+O\left(u^{2}\right)\right]^{\alpha-1}(1-u)^{\beta-1}}{\alpha u^{\alpha-1} \Gamma(\alpha)}=\frac{\beta^{\alpha}}{\alpha \Gamma(\alpha)} .
$$


Hence $H_{2}(u) \sim a_{1} u^{\alpha}$ as $u \rightarrow 0+$, where $a_{1}=\beta^{\alpha} /[\alpha \Gamma(\alpha)]$. It is regularly varying with index $\alpha$ at $u=0+$, and its inverse function $H_{2}^{-1}(u)$ is regularly varying with index $1 / \alpha$. Let $\ell_{H_{2}^{-1}}$ be the slowly varying function associated with $H_{2}^{-1}$. If $C(u, u)$ is of index $\kappa_{L}$ at $u=0+$, then

$$
\begin{aligned}
& H_{2}\left(C\left(H_{2}^{-1}(u), H_{2}^{-1}(u)\right)\right) \sim a_{1}\left[C\left(H_{2}^{-1}(u), H_{2}^{-1}(u)\right)\right]^{\alpha} \\
\sim & \left.\left.a_{1}\left[H_{2}^{-1}(u)\right]^{\kappa_{L}} \ell\left(H_{2}^{-1}(u)\right)\right]^{\alpha} \sim a_{1} u^{\kappa_{L}}\left[\ell_{H_{2}^{-1}}(u)\right) \ell\left(H_{2}^{-1}(u)\right)\right]^{\alpha}
\end{aligned}
$$

as $u \rightarrow 0+$. By Theorem A2 in the appendix, $\left.\left[\ell_{H_{2}^{-1}}(u)\right) \ell\left(H_{2}^{-1}(u)\right)\right]^{\alpha}$ is slowly varying. Thus, $\kappa_{L, H_{2}}=\kappa_{L}$. For the upper tail order, similar to the arguments for Equation (15), by L'Hopital Rule and the fact that $1-H_{2}(1-v)=H_{1}(v)$, we have that

$$
\begin{aligned}
& \lim _{v \rightarrow 0^{+}} \frac{H_{1}(1-C(1-v, 1-v))}{H_{1}(v)} \\
= & \lim _{v \rightarrow 0^{+}}\left[\frac{-\log (1-C(1-v, 1-v))}{-\log (v)}\right]^{\alpha-1}\left[\frac{1-C(1-v, 1-v)}{v}\right]^{\beta-1} C^{\prime}(1-v, 1-v) \\
= & \lim _{v \rightarrow 0^{+}}\left[2-\frac{\bar{C}(1-v, 1-v)}{v}\right]^{\beta}
\end{aligned}
$$

since $\lim _{v \rightarrow 0+} \log (2 v-\bar{C}(1-v, 1-v)) /-\log (v)=1$ and $\lambda_{U}=2-\lim _{v \rightarrow 0+} C^{\prime}(1-v, 1-v)$. Therefore,

$$
\begin{aligned}
& \lim _{u \rightarrow 0^{+}} \frac{\bar{C}_{H_{2}}(1-u, 1-u)}{u}=\lim _{v \rightarrow 0^{+}} \frac{\bar{C}_{H_{2}}\left(H_{2}(1-v), H_{1}(1-v)\right)}{1-H_{2}(1-v)} \\
= & 2-\lim _{v \rightarrow 0^{+}} \frac{1-H_{2}(C(1-v, 1-v))}{1-H_{2}(1-v)}=2-\lim _{v \rightarrow 0^{+}}\left[2-\frac{\bar{C}(1-v, 1-v)}{v}\right]^{\beta}
\end{aligned}
$$

that is finite if the upper tail dependence of the initial copula is finite. If $\kappa_{U}=1$, Equation (19) equals to $2-\left[\left(2-\lambda_{U}\right)\right]^{\beta}$. If $\kappa_{U}>1$, then $\lambda_{U}=0$ and Equation (19) equals to $2-2^{\beta}$.

In summary, the new families of copulas generated by the UGI and UGII distortions have an upper tail order of 1 and the same lower tail order as the initial copula.

According to the results in Section 2 and Section 3, Table 1 tabulates the upper and lower tail dependence coefficients of the three initial copulas considered as examples in this paper and those of the distorted copulas. The distorted Clayton copulas allow one to capture both lower and upper tail dependence. The Gumbel copula ability to model strong upper tail dependence is retained after distortion. The Frank copula has neither lower nor upper tail dependence. However, the distorted Frank copula can accommodate data with upper tail dependence.

\section{An Empirical Application}

In this section, we apply the proposed copula families to the economic indicator data ascertained from the Trading Economics website (https://tradingeconomics.com). The main purpose is not to address the economic implications but to examine how the new families of copulas perform when fit to the data.

The data contain the gross domestic product (GDP) in billion US dollars and the government debt to GDP reported by more than 173 countries in 2017. Countries with missing values are 
Table 1: Tail dependence coefficients of distorted copulas with initial Clayton, Gumbel and Frank copulas.

\begin{tabular}{lcccccc}
\hline Copula & $\lambda_{L}$ & $\lambda_{U}$ & $\lambda_{L, H_{1}}$ & $\lambda_{U, H_{1}}$ & $\lambda_{L, H_{2}}$ & $\lambda_{U, H_{2}}$ \\
\hline Clayton & $2^{-1 / \theta}$ & $\kappa_{U}=2$ & $2^{-\beta / \theta}$ & $2-2^{\alpha}$ & $2^{-\alpha / \theta}$ & $2-2^{\beta}$ \\
Gumbel & $\kappa_{L}=2^{1 / \theta}$ & $2-2^{1 / \theta}$ & 0 & $2-2^{\alpha / \theta}$ & 0 & $2-2^{\beta / \theta}$ \\
Frank & $\kappa_{L}=2$ & $\kappa_{U}=2$ & 0 & $2-2^{\alpha}$ & 0 & $2-2^{\beta}$ \\
\hline
\end{tabular}

Table 2: Summary statistics for the data, where $\mathrm{SD}=$ standard deviation.

\begin{tabular}{lrrrrrrr}
\hline Variables & $n$ & mean & SD & median & min & max & range \\
\hline Debt to GDP & 173 & 53.31 & 33.44 & 46.40 & 2.80 & 253 & 250.2 \\
GDP & 173 & 633.79 & 2410.00 & 48.72 & 0.39 & 19391 & 19390.2 \\
\hline
\end{tabular}
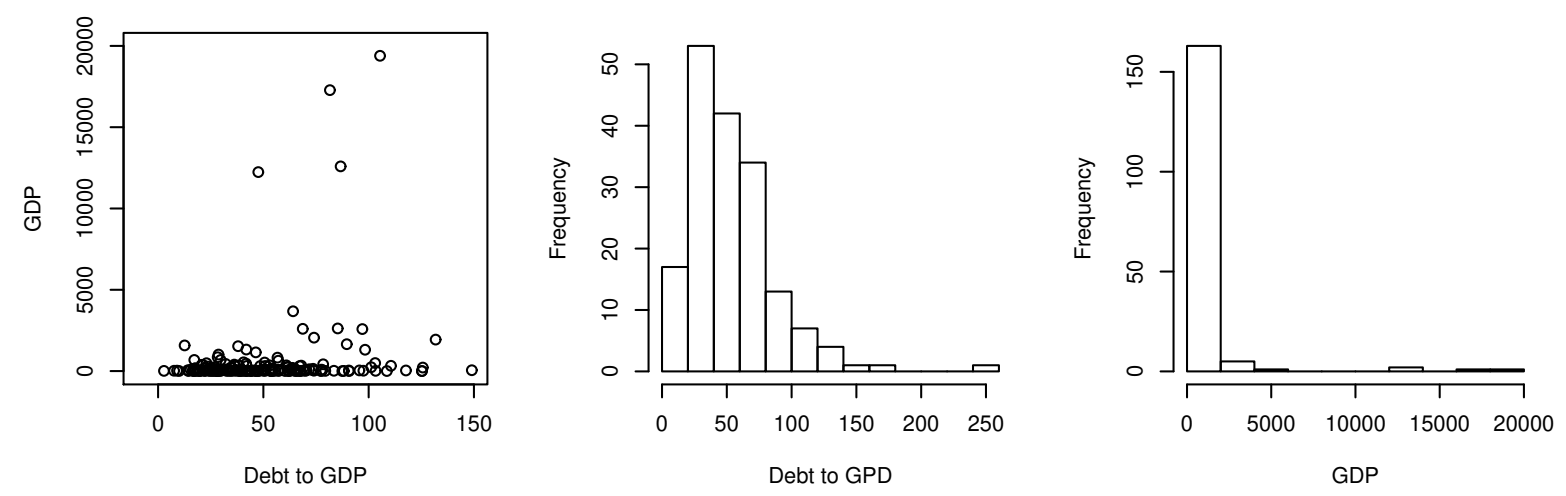

Figure 3: Scatter plot and histograms for GDP and Debt to GDP.

deleted. Basic numerical and graphical summaries of the two variables GDP $(X)$ and Debt to GDP $(Y)$ are shown below.

The semiparametric pseudo-maximum-likelihood approach (Genest et al., 1995) is used to estimate the parameters. The observations $\left(X_{i}, Y_{i}\right)$ are first converted into pseudo-observations $\left(U_{i}, V_{i}\right)$ by component-wise applying the empirical distribution functions to the data and scaling the result by $n /(n+1)$, where $n$ is the sample size. The parameter estimates are then obtained by minimizing the pseudo $\log$-likelihood $\sum_{i=1}^{n} \log c\left(U_{i}, V_{i}\right)$, where $c(u, v)$ is the copula density function.

In addition to the Clayton, Frank and Gumbel copulas, we fit the beta, UGI and UGII distorted copulas using the mle() function in R. The parameters of the admissible beta distribution are constrained such that $\alpha \geq 1$ and $0<\beta \leq 1$. function. The parameter $\alpha$ in the UGI distortion and the parameter $\beta$ in the beta and UGII distortions are constrained to the interval $(0,1]$. In these cases, a monotone and invertible re-parameterization of $\alpha=e^{\alpha_{1}} /\left(1+e^{\alpha_{1}}\right)$ or $\beta=e^{\beta_{1}} /\left(1+e^{\beta_{1}}\right)$ is implemented to avoid possible optimization problems. Therefore, estimates 
Table 3: Pseudo-likelihood estimation results with standard errors in parentheses.

\begin{tabular}{lrrrrrrr}
\hline Copula & $\widehat{\theta}$ & $\widehat{\alpha}_{1}$ & $\widehat{\beta}$ & PLL & $\widehat{\tau}$ & $\widehat{\lambda}_{L}$ & $\widehat{\lambda}_{U}$ \\
\hline Clayton & $0.06(0.10)$ & - & - & 0.17 & 0.027 & 0.00 & 0.00 \\
Frank & $0.71(0.47)$ & - & - & 1.13 & 0.079 & 0.00 & 0.00 \\
Gumbel & $1.12(0.06)$ & - & - & 2.98 & 0.103 & 0.00 & 0.14 \\
UG1-Frank & $-0.54(0.71)$ & $1.46(0.45)$ & $1.00(0.00)$ & 3.794 & 0.075 & 0.00 & 0.25 \\
UG1-Gumbel & $0.86(0.17)$ & $0.82(0.87)$ & - & 3.766 & 0.077 & 0.00 & 0.46 \\
UGII-Frank & $-0.50(0.93)$ & $1.05(0.98)$ & $1.54(1.91)$ & 3.797 & 0.075 & 0.00 & 0.23 \\
UGII-Gumbel & $0.87(0.32)$ & $1.14(1.41)$ & $1.02(1.00)$ & 3.758 & 0.079 & 0.00 & 0.34 \\
Beta-Frank & $-0.47(0.68)$ & $1.00(0.00)$ & $1.46(0.46)$ & 3.795 & 0.075 & 0.00 & 0.25 \\
Beta-Gumbel & $0.89(0.16)$ & - & $0.98(0.89)$ & 3.750 & 0.078 & 0.00 & 0.24 \\
\hline
\end{tabular}

of $\alpha_{1}$ and $\beta_{1}$ are reported. For the beta-Frank model, Table 3 reports the maximized pseudo-loglikelihood values (PLL) and the parameter estimates with their standard error in parentheses. We also computed the estimated Kendall's tau coefficients using the estimates $\widehat{\theta}, \widehat{\alpha}$ and $\widehat{\beta}$ to estimate the tail dependence coefficients using Table 1. For beta-Frank model, the maximum PLL is obtained at $\alpha=1$ and a standard error of zero is assigned.

The sample Pearson's correlation coefficient between the two variables is 0.23 , resulting in the conclusion that the correlation coefficient is significantly different from 0 with a p-value of 0.002. The sample Kendall's tau coefficient is 0.074 , statistically insignificant with a p-value of 0.15. The UGII-Frank and UGI-Frank copula models perform better than the Clayton, Gumbel and Frank copulas for the data set in terms of PLL. The initial Frank copula may have offered the most flexibility as the $\theta$ value can be either positive or negative. While the sample Kendall's tau is not statistically significant, there seems to exist an upper tail dependence between GDP and Debt to GDP according to the best performers, the UGII-Frank and UGI-Frank copula models.

\section{Conclusions}

The purpose of this paper has been to introduce new families of copulas based on distributional distortions. Specifically, unit gamma distortions are proposed to be applied to existing copulas. Issues such as extensions to multivariate copulas and estimation of parameters need further investigation.

This paper offers the idea that a cumulative distribution function with support of $[0,1]$ can be employed in the right-composition rule. The framework in Equation (4) can be employed to generate new families of bivariate joint distributions, which entails new families of bivariate copulas. The addition of parameters allows more flexibility and hence better fit to data as seen in the empirical example. When the copula being distorted is Archimedean, the distributional distortion can be seen as a method to construct a new Archimedean generator by applying the right-composition rule.

Kendall's tau coefficients are derived for the cases when the initial copulas are Clayton, Gumbel and Frank copulas. They may not have closed forms but can be numerically computed using Equation (10) or (20). Note that Spearman's rank correlations can be computed by using the numerical integration formula given by $12 \int_{[0,1] \times[0,1]} C(u, v) d u d v-3$ or $3-12 \int_{[0,1] \times[0,1]} u C_{2 \mid 1}(v \mid u) d u d v$. The impact of the distortions on Kendall's tau coefficient is demonstrated via three-dimension 
Table 4: Generators, lambda functions, and Kendall's tau coefficients of the Clayton, Gumbel and Frank copulas.

\begin{tabular}{lccc}
\hline Copula & $\phi(\cdot)$ & $\lambda(\cdot)$ & $\tau$ \\
\hline Clayton & $\frac{1}{\theta}\left(t^{-\theta}-1\right), \theta \geq 0$ & $\left(t^{\theta+1}-t\right) / \theta$ & $\frac{\theta}{\theta+2}$ \\
Gumbel & $(-\log (t))^{\theta}, \theta \geq 1$ & $t \log (t) / \theta$ & $1-\theta^{-1}$ \\
Frank & $-\log \left(\frac{e^{-\theta t}-1}{e^{-\theta}-1}\right), \theta \neq 0$ & $\frac{1}{\theta} \ln \frac{e^{-\theta t}-1}{e^{-\theta}-1}\left(e^{\theta t}-1\right)$ & Equation (11) \\
\hline
\end{tabular}

plots of Kendall's tau for the cases when the initial copulas are the Clayton, Gumbel and Frank copulas.

Results on tail dependence coefficients are obtained for the induced copulas. The induced copulas can accommodate a wider range of upper tail dependence coefficient values. For example, the upper tail dependence coefficient of the distorted Frank copula ranges from 0 to $2-2^{\beta}$, while the Frank copula has zero upper tail dependence. For the property of the tail order, in summary, the new families of copulas generated by the UGI and UGII distortions both have an upper tail order of 1 and a lower tail order of the initial copula.

\section{Appendix: Preliminaries}

The preliminaries for the properties studied in this article are staged in this appendix.

\section{Kendall's Tau Coefficient}

Kendall's tau concordance coefficient between two continuous variables is the probability of concordance minus the probability of discordance. For two continuous random variables with a copula $C$, Kendall's tau, denoted by $\tau$, between the two variables can be calculated by

$$
\tau=1-4 \int_{0}^{1} \int_{0}^{1} \frac{\partial C}{\partial u}(u, v) \frac{\partial C}{\partial v}(u, v) d u d v .
$$

Genest and Rivest (1993) show that Kendall's function $K(t)=P(C(u, v) \leq t)$ of an Archimedean copula with strict generator $\phi(\cdot)$ can be expressed as $K(t)=t-\phi(t) / \phi^{\prime}(t)$ and that

$$
\tau=3-4 \int_{0}^{1} K(t) d t=1+4 \int_{0}^{1} \lambda(t) d t
$$

where the lambda function $\lambda:[0,1] \rightarrow[-1,0]$ is defined to be $\phi(t) / \phi^{\prime}(t)$. Table 4 displays the generator $\phi(\cdot)$, lambda function and $\tau$ of the copulas used as examples in this paper.

\section{Tail Dependence coefficients}

A tail dependence coefficient measures the probability that a random variable $X$ reaches extreme values given another variable $Y$ attains extreme values. The upper tail dependence coefficient 
$\lambda_{U}$ is the limit, if exists, defined to be

$$
\lambda_{U}=\lim _{v \rightarrow 1-} P\left(X>F^{-1}(v) \mid Y>F^{-1}(v)\right)>0
$$

The joint survival function is $\bar{C}(u, v)=P(U>u, V>v)=1-u-v+C(u, v)$. If the limits exist, then the upper tail dependence coefficient $\lambda_{U}$ and the lower tail dependence coefficient $\lambda_{L}$ are

$$
\begin{aligned}
& \lambda_{U}=\lim _{u \rightarrow 1-} \frac{\bar{C}(u, u)}{1-u}=2-\lim _{u \rightarrow 1^{-}} \frac{1-C(u, u)}{1-u}=2-\lim _{u \rightarrow 1^{-}} \frac{d C(u, u)}{d u} \\
& \lambda_{L}=\lim _{u \rightarrow 0^{+}} \frac{C(u, u)}{u}=\lim _{u \rightarrow 0^{+}} \frac{d C(u, u)}{d u}
\end{aligned}
$$

If $\lambda_{U}$ is in $(0,1]$, then $C$ has upper tail dependence. If it is 0 , then $C$ has no upper tail dependence. Similar conclusions are made for $\lambda_{L}$.

\section{Tail Orders}

We next describe the concepts of tail order and tail order functions that Hua and Joe (2011) propose as an integrated way to study the strength of dependence in the joint tails; see also Joe (2014).

For a bivariate copula $C$, if $C(u, u) \sim u^{\kappa_{L}} \ell(u)$ as $u \rightarrow 0^{+}$, then $\kappa_{L}$ is referred to as the lower tail order of the copula $C$, where $\ell_{C}(u)$ is slowly varying at $0+$. Let $\widehat{C}(u, v)=\bar{C}(1-u, 1-v)=$ $u+v-1+C(1-u, 1-v)$ be the survival copula. The upper tail order is defined as $\kappa_{U}$ if $\widehat{C}(u, u) \sim u^{\kappa_{U}} \ell_{\widehat{C}}(u)$ as $u \rightarrow 0^{+}$for some slowly varying function $\ell_{\widehat{C}}(u)$ at $0+$. When $\kappa_{L}=2$ and $\ell_{C}(u) \rightarrow a$ as $u \rightarrow 0^{+}$, for some positive $a$, the bivariates are near independent in the lower tail. If $1<\kappa_{L}<2$, the bivariates are positively associated and have intermediate tail dependence. The case $\kappa_{L}=1$ corresponds to the usual tail dependence $\lambda_{L} \in(0,1)$ defined to be $\lim _{u \rightarrow 0+} C(u, u) / u=\lim _{u \rightarrow 0+} \ell_{L}(u)$. Similar conclusions are made for $\kappa_{U}$.

Assume that a bivariate copula $C$ has a lower tail order of $\kappa_{L}$ and an upper tail order of $\kappa_{U}$, then the respective lower tail and upper tail order functions of $C$ are given by, if the limits exist, for $0<w_{1}, w_{2}<\infty$,

$$
b\left(w_{1}, w_{2}\right)=\lim _{u \rightarrow 0^{+}} \frac{C\left(u w_{1}, u w_{2}\right)}{u^{\kappa_{L}} \ell_{C}(u)} \text { and } b^{*}\left(w_{1}, w_{2}\right)=\lim _{u \rightarrow 1^{-}} \frac{\widehat{C}\left(u w_{1}, u w_{2}\right)}{u^{\kappa_{U} \ell_{\widehat{C}}}(u)} .
$$

\section{Regular Variation}

A brief account on properties of regularly varying functions is submitted here since the properties are needed here. Proofs and details can be found in Bingham et al. (1987).

Let $f_{1}(x) \sim f_{2}(x)$ denote that the real-valued functions $f_{1}$ and $f_{2}$ are asymptotically equal such that $\lim _{x} f_{1}(x) / f_{2}(x) \rightarrow 1$ as $x \rightarrow 0$ (or $\infty$ ). A positive measurable function $f$ defined on some $[a, \infty)$ for some $a>0$ is said to be regularly varying with index $\rho$, and we write $f \in \mathcal{R}(\rho)$, if for some real number $\rho$ it satisfies

$$
\lim _{x \rightarrow 0^{+}} f(\gamma x) / f(x)=\gamma^{\rho} \text { for all } \gamma>0,
$$

The function $f$ is said to be slowly varying if $\rho=0$.

Theorem A1 (Karamata's Characterization Theorem). Every regularly varying function $f$ with index $\rho$ is of the form $f(x)=x^{\rho} \ell(x)$, where $\ell$ is a slowly varying function. 
Theorem A2 (Closure Property). Suppose that $f_{1} \in \mathcal{R}\left(\rho_{1}\right)$ and $f_{2} \in \mathcal{R}\left(\rho_{2}\right)$. Then (i) $f_{1}^{\rho} \in$ $\mathcal{R}\left(\rho \rho_{1}\right)$. (ii) $f_{1} \circ f_{2} \in \mathcal{R}\left(\rho_{1} \rho_{2}\right)$. (iii) $f_{1}+f_{2} \in \mathcal{R}(\rho)$, where $\rho=\max \left(\rho_{1}, \rho_{2}\right)$.

Theorem A3 (Karamata's Integral Theorem). If $\ell$ is slowing varying, for $\rho>-1$, then $\int_{c}^{x} t^{\rho} \ell(t) d t \sim x^{\rho+1} \ell(x) /(\rho+1)$, as $x \rightarrow 0($ or $\infty)$.

Theorem A4. If $f_{1} \in \mathcal{R}(\rho)$ with $\rho>0$, there exists $f_{2} \in R(1 / \rho)$ such that $f_{1}\left(f_{2}(x)\right) \sim$ $f_{2}\left(f_{1}(x)\right) \sim x$ as $x \rightarrow 0$. Here $f_{2}$, an asymptotic inverse of $f_{1}$, is determined uniquely to within asymptotic equivalence.

\section{References}

Alzaatreh A, Lee C, Famoye F (2013). A new method for generating families of continuous distributions. Metron, 71: 63-79.

Bingham NC, Goldie CM, Teugels JL (1987). Regular Variation. Cambridge University Press, Cambridge.

Di Bernardino E, Rullière D (2013). Distortions of multivariate distribution functions and associated level curves: Applications in multivariate risk theory. Insurance: Mathematics and Economics, 53: 190-205.

Di Bernardino E, Rullière D (2016). On tail dependence coefficients of transformed multivariate Archimedean copulas. Fuzzy Sets and Systems, 284: 89-112.

Eugene N, Lee C, Famoye F (2010). Bet-normal distribution and its applications. Communications in Statistics-Theory and Methods, 31: 497-512.

Fischer M, Köck C (2010). Constructing and generalizing given multivariate copulas: A unifying approach. Statistics, 46: 1-12.

Genest C, Ghoudi K, Rivest LP (1995). A semi-parametric estimation procedure of dependence parameters in multivariate families of distributions. Biometrika, 82: 543-552.

Genest C, Ghoudi K, Rivest LP (1998). Discussion on the "Understanding relationships using copulas" by Frees and Valdez. North American Actuarial Journal, 2: 143-149.

Genest C, Rivest LP (1993). Statistical inference procedures for bivariate Archimedean copulas. Journal of the American Statistical Association, 88: 1034-1043.

Grassia A (1977). On a family of distributions with argument between 0 and 1 obtained by transformation of the Gamma distribution and derived compound distributions. Australian Journal of Statistics, 19: 108-114.

Hua L, Joe H (2011). Tail order and intermediate tail dependence of multivariate copulas. Journal of Multivariate Analysis, 102: 1454-1471.

Joe H (2014). Dependence Modeling with Copulas. Chapman and Hall/CRC, 1st edition.

Morillas P (2005). A method to obtain new copulas from a given one. Metrika, 61: 169-184.

Nadarajah S, Rocha R (2016). Newdistns: An R package for new families of distributions. Journal of Statistical Software, 69: 1-32.

Nelsen RB (2006). An Introduction to Copulas. Springer, New York.

Samanthi RGM, Sepanski J (2019). A bivariate extension of the beta generated distribution derived from copulas. Communications in Statistics-Theory and Methods, 48: 1043-1059.

Sklar A (1959). Fonctions de répartition à n dimensions et leurs marges. Publications de l'Institut Statistique de l'Université de Paris, 8: 229-231.

Valdez E, Xiao Y (2011). On the distortion of a copula and its margins. Scandinavian Actuarial Journal, 4: 292-317. 
Wirch J, Hardy M (1999). A synthesis of risk measures for capital adequacy. Insurance: Mathematics and Economics, 25: 337-347. 